\title{
Bare Lymphocyte Syndrome

\author{
Consequences of Absent Class II Major Histocompatibility Antigen Expression \\ for B Lymphocyte Differentiation and Function
}

Loran T. Clement, Susan Plaeger-Marshall, Albert Haas, Andrew Saxon, and Audrey M. Martin

Departments of Pediatrics and Medicine, University of California, Los Angeles School of Medicine, Los Angeles, California 90024

\begin{abstract}
The bare lymphocyte syndrome is a rare combined immunodeficiency disorder associated with the absence of class I and/or class II major histocompatibility (MHC) antigens. Although it has been inferred that the immune deficiency is a consequence of disordered MHC-restricted interactions among otherwise normal cells, the biological capabilities and differentiation of $B$ lymphocytes deficient in class II MHC antigens have not been rigorously analyzed. We have examined the phenotypic and functional attributes of B cells with absent class II MHC antigens. Our data demonstrate that these $B$ cells are intrinsically defective in their responses to membrane-mediated activation stimuli. In addition, virtually all the $B$ cells had phenotypic evidence of arrested differentiation at an immature stage. Finally, these $B$ cells also failed to express the C3d-EBV receptor normally present on all $B$ lymphocytes. These data indicate that class II MHC molecules are vital participants in early events of the $B$ cell activation cascade, and that other nonMHC membrane molecules may also be absent as a consequence of either arrested differentiation or as a result of the basic defect affecting the expression of MHC membrane antigens.
\end{abstract}

\section{Introduction}

The bare lymphocyte syndrome (BLS) ${ }^{1}$ is a rare immunodeficiency disease caused by or associated with the failure of expression of cell surface antigens encoded for by the major histocompatibility complex (MHC). Since the initial descriptions of this disorder, approximately 30 patients with this disorder have been described (1-5). It is now apparent that this syndrome is heterogeneous with regard to defective cell surface antigen expression. In some patients with this disorder, there is defective expression of only class I MHC antigens encoded for by the HLA-A, B, and C genetic loci, while in other patients, class II MHC antigens (HLA-DR, DQ, and DP antigens) are not expressed $(4,5)$. Patients who fail to express both class I

Address reprint requests to Dr. Clement, Department of Pediatrics, UCLA School of Medicine, Los Angeles, CA 90024.

Received for publication 15 June 1987 and in revised form $24 \mathrm{Au}$ gust 1987.

1. Abbreviations used in this paper: BCGF, B cell growth factors; BLS, bare lymphocyte syndrome; PE, phycoerythrin; SAC, Staphylococcus aureus, Cowan I strain; $\left[{ }^{3} \mathrm{H}\right] \mathrm{TdR}$, tritiated thymidine.

J. Clin. Invest.

(c) The American Society for Clinical Investigation, Inc.

0021-9738/88/03/0669/07 \$2.00

Volume 81, March 1988, 669-675 and II MHC antigens have also been identified (5). Clinically, this syndrome is manifest as a combined immunodeficiency presenting early in life, and affected individuals are susceptible to a number of severe and/or opportunistic infections by a wide variety of pathogens. Chronic, severe diarrhea and malabsorption is also a characteristic feature of patients with the BLS, and death is common within the first few years of life.

The intrinsic molecular lesions responsible for the defective expression of membrane MHC antigens in this syndrome have not yet been identified. Similarly, the pathogenetic basis for the defects in cellular and humoral immunity has only inferentially been defined. Because MHC antigens have an important role as recognition structures for interactions among immunocompetent cells (6-8), it has generally been assumed that the immune deficiency can be attributed to the inability of cells to recognize antigens in the context of self MHC molecules. However, there are other possible mechanisms that could contribute to the defective immune responsiveness seen in individuals with the BLS. For example, recent evidence suggests that the functions of class II MHC molecules in lymphocyte responses are complex and are not restricted to mediating cell-cell interactions. Class II MHC antigens appear to be directly involved in B cell activation events that are independent of interactions between immunocompetent cells (9-12). Similarly, defective B cell differentiation may also lead to immune deficiency. At present, information concerning the differentiation of B cells in the BLS is scanty.

We have previously shown that antibodies reactive with class II MHC antigens inhibit B cell activation (11). However, these studies could not determine whether this inhibitory effect was the result of a direct negative signal produced by the bound antibody, or whether the inhibition was a consequence of disrupted function and/or intracellular transit of class II molecules. Because B cells in the class II MHC deficiency syndrome do not express these molecules, this "experiment of nature" offers the opportunity to distinguish between these two possible mechanisms. In this study, we have examined the phenotypic and functional characteristics of B lymphocytes in a patient with the class II MHC deficiency syndrome. Our data demonstrate that B cells that do not express class II antigens are intrinsically defective in their responses to inductive membrane-mediated activation stimuli. In addition, virtually all the B cells had phenotypic evidence of arrested differentiation at an immature stage characterized by expression of the HB4 antigen. Finally, these $B$ cells failed to express the C3d-EBV receptor normally present on circulating B lymphocytes. These data demonstrate the intimate involvement of class II MHC antigens in the intracellular events required for B cell activation. Furthermore, they show that the defect(s) responsible for this syndrome may also affect the expression of nonMHC encoded membrane molecules. 


\section{Methods}

Patient and control subjects. The individual with the bare lymphocyte syndrome was a male born to nonconsanguinous parents. He initially presented with failure to thrive, diarrhea, and thrush at 4 mo of age. Initial evaluation revealed panhypogammaglobulinemia, normal numbers of circulating $T$ and $B$ lymphocytes, but an absence of cells expressing HLA-DR antigens or other class II MHC antigens (13). The functional capabilities and phenotypic characteristics of the patient's $B$ cells were examined serially over an 11-mo span and did not vary significantly during this period. Control cell populations were obtained from age-matched normal individuals or, when appropriate, from healthy adult volunteers. Informed consent was obtained under protocols approved by the Human Subjects Protection Committee of the UCLA School of Medicine.

Monoclonal antibodies and reagents. Monoclonal antibodies (mab) used in these studies include: OKT4, an anti-CD4 mab, and Genox 3.53 , an antibody reactive with HLA-DQ antigens (obtained from the American Type Culture Collection, Rockville, MD); 2D2, a mab analogous to the Leu-2 mab and reactive with CD8+ cells (14); HB-4, a mab reactive with a subpopulation of $B$ cells in peripheral blood and bone marrow (15); HB-5, which reacts with the CD21 antigen that serves as the $B$ cell $C R 2$ receptor for C3d and Epstein-Barr virus (C3dEBV) (16); and HB-7 (Leu-17), which reacts with the CD38 antigen present on immature cells of B lymphocyte lineage (17). Another CD38 mab, OKT10, as well as OKT9, which reacts with the transferrin receptor, were obtained from Ortho Pharmaceuticals (Raritan, NJ). The Leu-1 mab, the Leu-10 (anti-DQ) mab, and antibodies to HLADR antigens and the IL-2 receptor were obtained from Becton-Dickinson (Sunnyvale, CA). The B7/21 (anti-HLA-DP) and LB3.1 (antiHLA-DR) mab were kindly provided by Dr. Michael Brenner (DanaFarber Cancer Institute). The $\mathrm{Ba} 1$ and $\mathrm{Ba} 2$ mab were obtained through the generosity of Drs. Tucker LeBien and John Kersey. Affinity-purified, fluorochrome-conjugated goat antibodies specific for human or murine immunoglobulin heavy or light chains were obtained from Southern Biotechnology Associates (Birmingham, AL).

Preparation and fractionation of mononuclear cell populations. Methods for the preparation and fractionation of cells have been described in detail (14). In brief, MNC were isolated from heparinized venous blood samples by Ficoll-Hypaque density gradient centrifugation. After incubation on plastic culture dishes to deplete monocytes, T lymphocytes were isolated by rosette formation with sheep erythrocytes treated with 2-aminoethylisothiouronium bromide and density gradient centrifugation. In most experiments, the nonrosetting (E-) cells were used as the source of B lymphocytes. Methods used to isolate small resting B cells from the E- fraction for other experiments are described below.

Media. All cells were washed and cultured in RPMI 1640 medium supplemented with $10 \%$ fetal calf serum (FCS; Gibco Laboratories, Grand Island, NY), $2 \mathrm{mM}$ glutamine, penicillin $(100 \mathrm{U} / \mathrm{ml})$, and streptomycin $(0.1 \mathrm{mg} / \mathrm{ml})$.

Purification of $T$ cell subpopulations. The CD4+ subpopulation of $\mathrm{T}$ cells was isolated by negative-selection panning methods, as described (18). In brief, petri dishes were coated with affinity-purified goat anti-mouse immunoglobulin antibodies (Southern Biotechnology Associates). T cells were treated with the 2D2 (CD8) mab, washed, and sequentially incubated on two antibody-coated plates for $1 \mathrm{~h} \mathrm{at} 4^{\circ} \mathrm{C}$ to remove $\mathrm{CD} 8+$ cells. The nonadherent cells isolated in this fashion were $>88 \%$ CD4t.

Immunofluorescent phenotypic analysis. Methods for direct and indirect immunofluorescent analysis of cell membrane antigens have been described in detail $(11,16)$. Cells were stained either directly with rhodamine, fluorescein (FITC)- or phycoerythrin (PE)-labeled antibodies, or indirectly with unconjugated mab followed by fluorochrome-conjugated goat anti-mouse Ig antibodies. For one-color indirect staining experiments, FITC-conjugated goat anti-mouse Ig (Southern Biotechnology Associates) was used as the second layer. In other experiments, the appropriate combination of fluorochromeconjugated goat antibodies specific for murine heavy chain isotypes were used. Cells stained with FITC only, PE only, or with both FITC and PE were analyzed by flow cytometry, using an EPICS C cytometer (Coulter Instruments, Inc., Hialeah, FL). For analyses of cells stained with rhodamine-conjugated antibodies (either alone or in combination with FITC), reactivity was assessed using a fluorescence microscope (E. Leitz, Inc., Rockleigh, NJ) with epi-illumination.

Evaluation of B lymphocyte size. To evaluate changes in B cell volume after stimulation with anti-mu antibodies, small resting $B$ cells were isolated using a unit gravity density gradient sedimentation technique (19). The separation apparatus used consisted of a cylindrical incubation chamber mounted on a variable angle platform. An inletoutlet valve attached to a gradient maker and a peristaltic pump was located at the bottom of the chamber. For gradient preparation, the chamber was placed at a $30^{\circ}$ angle from horizontal, and $900 \mathrm{ml}$ of a continuous 2-4\% Ficoll gradient (in media containing 1\% FCS) was pumped into the bottom of the chamber. This was underlayered with $180 \mathrm{ml}$ of a $10 \%$ Ficoll cushion. Nonadherent T cell-depleted (E-) MNC suspended in $50 \mathrm{ml}$ of media containing $1 \%$ Ficoll were added to the top of the chamber, which was then returned to the horizontal position, and cells were allowed to sediment for $3 \mathrm{~h}$. The chamber was then repositioned at a $30^{\circ}$ angle from horizontal, and $30 \mathrm{ml}$ fractions were pumped from the bottom of the chamber. The size of the cells in each fraction was analyzed with a Coulter Channelyzer, and the fractions with small B lymphocytes were pooled. B cells isolated in this fashion are homogeneous in size (see Fig. 2), contain fewer than $0.1 \%$ esterase-positive monocytes, and have proliferative responses characteristic of small resting B cells (19). These cells were then cultured for $24 \mathrm{~h}$ in the presence of either a control antibody or monoclonal anti$\mathrm{mu}$ antibodies $(50 \mu \mathrm{g} / \mathrm{ml})$, and cell size changes were assessed with a Coulter Channelyzer.

$B$ cell proliferation assays. B cell proliferation was assessed in $\left[{ }^{3} \mathrm{H}\right]-$ thymidine $\left(\left[{ }^{3} \mathrm{H}\right] \mathrm{TdR}\right)$ assays (11). In most experiments, nonadherent E-cells were used as the source of B lymphocytes. The E- cells $\left(1 \times 10^{5}\right)$ were cultured in triplicate flat-bottomed microtiter wells in a total volume of $0.2 \mathrm{ml} /$ well. To assess $\mathrm{T}$ cell-independent $\mathrm{B}$ cell proliferation, cells were stimulated with SADA.4 $(50 \mu \mathrm{g} / \mathrm{ml})$, a mitogenic mab reactive with human mu heavy chains (20) or with formalin-fixed, protein-A bearing $S$. aureus (SAC) at a final concentration of $0.01 \%$. Controls included medium alone and medium containing an equivalent concentration of an unreactive mab. In addition, $T$ cell-dependent B proliferation was assessed, using a previously described method in which B cells are co-cultured with mitomycin $C$-treated $T$ cells and PHA (21). Cultures were incubated at $37^{\circ} \mathrm{C}$ for $60 \mathrm{~h}$, then pulsed with [ $\left.{ }^{3} \mathrm{H}\right] \mathrm{TdR}(0.5 \mu \mathrm{Ci} /$ well $)$. After an additional $14-16 \mathrm{~h}$ of culture, cells were harvested with a semiautomated harvester, and $\left[{ }^{3} \mathrm{H}\right] \mathrm{TdR}$ uptake was determined.

$B$ cell differentiation assays. The differentiation of $\mathbf{B}$ cells into antibody-secreting plasma cells was evaluated as previously described (22). Briefly, E- cells $\left(75 \times 10^{3}\right)$ were cultured in triplicate flat-bottom microtiter wells with varying numbers of $T$ cells or $T$ cell subpopulations and pokeweed mitogen (PWM; Gibco) at a final dilution of 1:250. After $7 \mathrm{~d}$, culture supernatants were harvested, and the IgG and IgM concentrations were measured using an ELISA. For the ELISA, microtiter ELISA plates (Dynatech, Alexandria, VA) were coated overnight with affinity-purified goat antibodies specific for either human mu or gamma heavy chains. After the plates were blocked with $1 \%$ bovine serum albumin, serial dilutions of the culture supernatants were incubated in the wells for $24 \mathrm{~h}$. The plates were then washed, and alkaline phosphatase-labeled goat anti-human Ig antibodies were added for $4 \mathrm{~h}$. The plates were again washed, and enzyme substrate was added for colorometric assessment of bound antibody, which was determined with a Dynatech ELISA plate reader. Background was determined in wells incubated with media alone. Quantitation of the IgG and IgM concentrations in the supernatants was made from a standard curve derived with Ig standards. 


\section{Results}

Phenotypic features of $B$ lymphocytes in BLS. During normal $B$ lymphocyte maturation, B-lineage cells undergo a sequence of changes in their expression of membrane antigens. Therefore, to assess the maturity of B cells in the BLS, we analyzed their membrane antigen phenotype by immunofluorescent staining with a panel of antibodies. Preliminary studies determined that $\sim 15-25 \%$ of blood nonadherent MNC expressed surface (sIg) molecules, the definitive marker for B cell identification. To determine the isotypic characteristics of the sIg present, two-color immunofluorescence analyses were performed. MNC from either BLS or normal controls were stained with rhodamine-conjugated goat antibodies specific for human Fab determinants (to detect all sIg + cells) and FITC-conjugated, affinity-purified goat antibodies specific for either mu, delta, alpha, or gamma heavy chains or for kappa or lambda light chains. As shown in Table I, virtually all sIg+ cells in the BLS expressed the mu and delta heavy chains, and the relative frequency of expression for each was only slightly higher than seen in controls. Only a small percentage $(\sim 3 \%)$ of the BLS cells had surface IgG molecules, and no sIgA+ cells were detected. The ratio of kappa/lambda light chain expression was $1.50-1.72$, which was normal. Both the absolute number and the relative frequency of $B$ lymphocytes among MNC in the BLS patient were normal for age. Thus, the absence of class II MHC antigens did not adversely affect B cell production, nor did it result in major qualitative alterations of surface Ig expression.

The expression of other cell membrane molecules on B lymphocytes was similarly analyzed. As shown in Table II, all MNC from this BLS patient expressed class I MHC antigens reactive with the W6/32 mab. Since the mean and peak fluorescence intensity were comparable for BLS and normal cells stained with this mab (data not shown), there did not appear to be any significant quantitative deficit of class I MHC antigens on any of the BLS cells. In contrast, no class II HLA-DR, DQ, or DP antigens were detectable on the BLS cells. Two-color immunofluorescence analyses demonstrated that virtually all of the B cells from both the control and BLS individuals ex-

Table I. Surface Immunoglobulin Expression by B Lymphocytes in the Bare Lymphocyte Syndrome

\begin{tabular}{lcc}
\hline \multicolumn{1}{c}{ Isotype* } & BLS $^{\ddagger}$ & Normal \\
\hline sIgM & 96 & $89-94$ \\
SIgD & 95 & $85-91$ \\
SIgG & 3 & $5-11$ \\
sIgA & 0 & $0-6$ \\
kappa/lambda & & \\
ratio & 1.61 & $1.40-1.72$ \\
\hline
\end{tabular}

\footnotetext{
* Nonadherent mononuclear cells were stained with FITC-labeled, affinity-purified goat anti-human Fab antibodies (to identify B cells) and with the appropriate rhodamine-labeled goat antibody specific for the indicated immunoglobulin isotype. The percent of Fab+ cells expressing each isotypic determinant was quantitated by fluorescence microscopy.

\# Mean percent of surface immunoglobulin-positive cells coexpressing the indicated chain in two experiments.
}

Table II. Membrane Antigens Expressed by B Lymphocytes in the Bare Lymphocyte Syndrome

\begin{tabular}{llcc}
\hline & & \multicolumn{2}{c}{$\begin{array}{c}\text { Percent of sIg + cells } \\
\text { coexpressing the antigen }\end{array}$} \\
\cline { 3 - 4 } \multicolumn{1}{c}{$\begin{array}{c}\text { Monoclonal } \\
\text { antibody* }\end{array}$} & \multicolumn{1}{c}{$\begin{array}{c}\text { Antigen } \\
\text { recognized }\end{array}$} & BLS & Normals \\
\hline W6/32 & Class I MHC & $97-99$ & $96-100$ \\
Anti-HLA-DR & HLA-DR & 0 & $>95$ \\
LB3.1 & HLA-DR & $0-1$ & $>95$ \\
Genox 3.53 & HLA-DQ & 0 & $>90$ \\
Leu-10 & HLA-DQ & 0 & $>90$ \\
B7/21 & HLA-DP & $0-1$ & $>90$ \\
B1 & CD20 & $95-97$ & $92-97$ \\
Leu-17 & CD38 & $65-71$ & $35-44$ \\
Leu-1 & CD5 & $0-2$ & $<2$ \\
HB-4 & B subset & $93-95$ & $54-62$ \\
HB-5 & CD21 & $3-5$ & $86-95$ \\
B2 & CD21 & $2-7$ & $86-95$ \\
& & & \\
\hline
\end{tabular}

\footnotetext{
* Nonadherent mononuclear cells were stained with FITC-labeled, affinity-purified goat anti-human Fab antibodies (to identify B cells) and with the indicated mab (either directly with PE-conjugated mab, or indirectly using the appropriate rhodamine-labeled goat antibody specific for the mab immunoglobulin isotype). The percent of Fab+ cells coexpressing each antigen was quantitated by fluorescence microscopy or flow cytometry.

₹ Percent of surface immunoglobulin-positive cells coexpressing the indicated antigen in two experiments.
}

pressed the B1 (CD20) antigen, whereas Leu-1+ (CD5+) B cells were virtually undetectable in each population. Expression of the Ba1, Ba2, and cALLa antigens on BLS and normal $B$ cells were comparable (data not shown). In contrast, the percentage of $B$ cells expressing the CD38 antigen, a molecule expressed by relatively immature B cells (17), was increased in the BLS. Similarly, whereas only $58 \%$ of control B cells expressed the HB-4 antigen, which is found on a subset of relatively immature circulating B cells (15), all sIgM+ B cells from the BLS patient coexpressed the HB-4 antigen. There was also a striking difference noted in the expression of the CD21 antigen, a molecule identified by the HB- 5 or B2 mabs that functions as the $B$ cell $C 3 d-E B V$ receptor $(23,24)$. Whereas almost all normal $B$ cells expressed this molecule, CD21 antigen expression by B cells in the BLS was virtually undetectable (Table II). The fluorescence staining intensity of anti-CD21 antibodies with B cell-enriched (E-) cells from normal or BLS individuals is quantitatively shown in Fig. 1.

Analysis of $B$ cell activation in $B L S$. Cross-linkage of sIgM molecules initiates a series of events that collectively are termed B cell activation. One early event that occurs with this stimulus is B cell enlargement $(25,26)$. To analyze the extent to which B cells deficient in class II MHC antigens could respond to signals provided by sIgM cross-linkage, small resting B cells were isolated from BLS or control MNC, and each population was stimulated with anti-mu antibodies at $37^{\circ} \mathrm{C}$. After $24 \mathrm{~h}, \mathrm{~B}$ cell size was analyzed with a Coulter Channelyzer. Results are shown in Fig. 2. Both normal B cells and those from the patient underwent significant enlargement after stimulation. The magnitude of the mean increase in cell volume was only slightly less for the B cells deficient in class II 


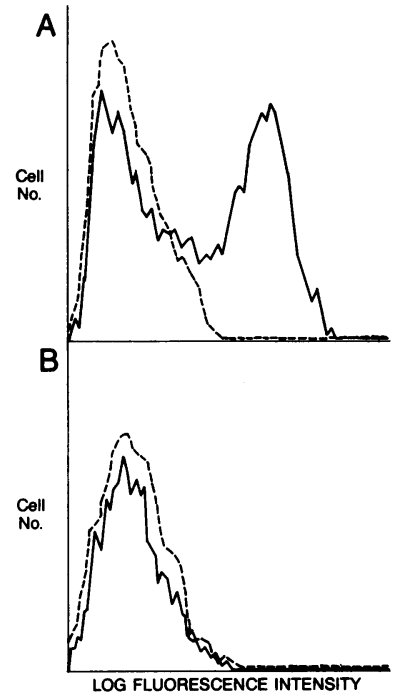

Figure 1. Absence of CD21 antigens (C3d/EBV receptors) in the bare lymphocyte syndrome. Nonadherent $T$ cell-depleted (E-) mononuclear cells from a normal control donor $(A)$ or from the bare lymphocyte syndrome patient $(B)$ were stained with either an unreactive control antibody (dotted line) or the HB-5 (anti-CD21) antibody (solid line) and analyzed by flow cytometry. The CD21 antigen was expressed by $53 \%$ of normal E- cells (which contained $56 \%$ sIgM + B cells) but was undetectable on cells from the bare lymphocyte syndrome patient (which contained $61 \%$ B cells).

antigens than was observed with normal B cells, and it was well within the normal range.

We also examined whether stimulation with anti-mu antibodies effected changes in the cell surface phenotype of the B lymphocytes. Anti-mu antibodies increased the relative amount of HLA-DR antigens expressed by normal B lymphocytes $\sim 300 \%$ (as estimated by changes in mean fluorescence staining intensity) but failed to induce detectable HLA-DR on the BLS cells (data not shown). Similarly, BLS cells treated with anti-mu antibodies did not express $B$ cell activation antigens such as transferrin receptors (as assessed with the OKT9 mab) or receptors for IL-2 (as assessed with an anti-IL 2 recep-

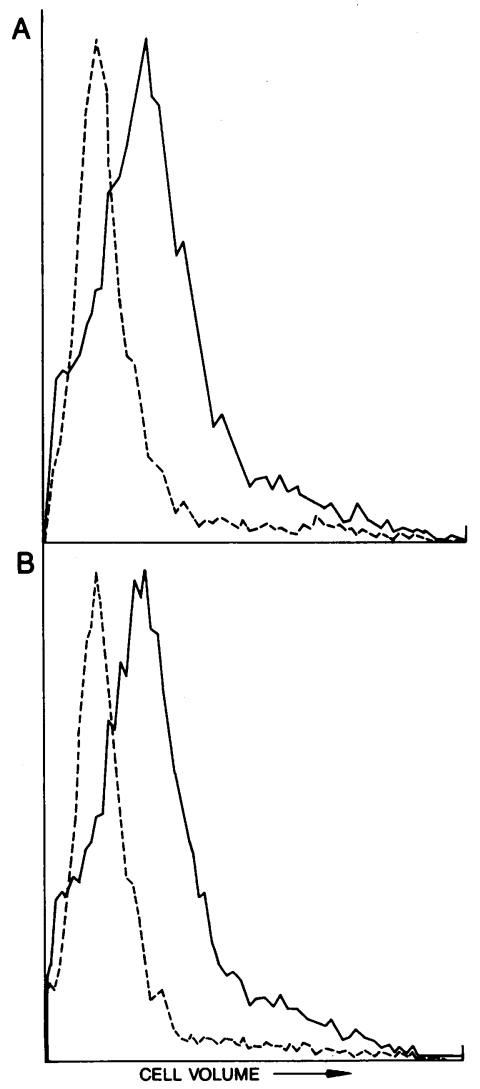

Figure 2. Effects of anti-mu stimulation on B cell size. Isolated small, resting B cells from a normal control $(A)$ or the patient with bare lymphocyte syndrome $(B)$ were cultured $24 \mathrm{~h}$ in either control medium (dotted line) or in the presence of anti-mu antibodies (solid line). Both normal B cells and those from the bare lymphocyte syndrome patient increased in size to a comparable degree. tor mab). Thus, although B cells deficient in class II MHC antigens can be induced to enlarge by cross-linkage of sIg molecules, no additional phenotypic evidence of $B$ cell activation was observed.

$B$ cell proliferation. Two assay systems were used to determine whether class II antigen-deficient B lymphocytes could be induced to proliferate by stimuli that are mitogenic for normal B cells. In the first series of experiments, B cells from normal or BLS individuals were stimulated with either antimu antibodies or with SAC, both of which act as T cell-independent mitogens. Representative data from one of three such experiments are shown in Table III. Whereas both of these stimuli induced significant proliferation in normal B cells, virtually no proliferation was observed in the B lymphocytes in the BLS. This was not due to suppressive effects, as the response of normal B cells was not inhibited (nor enhanced) by the inclusion of cells from the BLS individual (data not shown). Similarly, the unresponsiveness of BLS cells did not appear to be due to the absence of a requisite accessory cell function since the addition of control semiallogeneic adherent cells to BLS B cells did not confer responsiveness.

Because B lymphocytes can also be induced to proliferate by activated helper T cells (21), we analyzed whether B cells in the BLS could respond to mitogenic stimuli from activated autologous or allogeneic $T$ cells. In these experiments, normal or BLS B cells were cultured in the presence of PHA with purified autologous or allogeneic CD4+ $T$ cells (pretreated with mitomycin $C$ to abrogate their proliferative capability). As shown in Table IV, normal B cells underwent a vigorous proliferative response in the presence of activated CD4+ cells. Both the allogeneic CD4+ cells (i.e., those from the BLS patient) and the normal (autologous) $\mathrm{T}$ cells were able to induce this response. In contrast, B cells from the BLS patient were unresponsive to stimuli provided by either population of helper $T$ cells. When the response of these two B cell populations to preformed growth factors (BCGF) released by PWMactivated $T$ cells was analyzed, only the normal B cell population contained cells that responded to soluble factors. The defective response of the BLS B lymphocytes was again not corrected by adherent cells from the normal donor (not shown).

Despite numerous attempts at Epstein-Barr virus (EBV) transformation using a variety of approaches, including $T$ cell-depletion before EBV addition and/or culturing cells in cyclosporin $\mathrm{A}$, no evidence of proliferation or transformation

Table III. B Lymphocyte Proliferation in the Bare Lymphocyte Syndrome

\begin{tabular}{llr}
\hline & \multicolumn{2}{c}{$\left[{ }^{3} \mathrm{H}\right] \mathrm{TdR}$ uptake by B cells from: } \\
\cline { 2 - 3 } Stimulus* & \multicolumn{1}{c}{ BLS } & \multicolumn{1}{c}{ Normals } \\
\hline None & $800 \pm 37$ & $927 \pm 100$ \\
Anti-mu & $879 \pm 123$ & $10,296 \pm 593$ \\
SAC & $974 \pm 85$ & $21,233 \pm 854$ \\
BCGF & $673 \pm 122$ & $5,395 \pm 468$
\end{tabular}

* Nonadherent, T cell-depleted $(\mathrm{E}-)$ lymphocytes $\left(1 \times 10^{5} /\right.$ well $)$ were cultured with the indicated stimulus for $3 \mathrm{~d}$. Cultures were pulsed with $\left[{ }^{3} \mathrm{H}\right] \mathrm{TdR}$ during the final $14-16 \mathrm{~h}$ of culture. Results are expressed as mean counts per minute uptake \pm SEM. 
Table IV. T Cell-dependent B Lymphocyte Proliferation in the Bare Lymphocyte Syndrome

\begin{tabular}{lccc}
\hline & & \multicolumn{2}{c}{$\left[{ }^{3} \mathrm{H}\right] \mathrm{TdR}$ uptake } \\
\cline { 3 - 4 } \multicolumn{1}{c}{ T cells added* } & Mitogen & BLS & Normals \\
\hline None & - & $350 \pm 37$ & $427 \pm 144$ \\
None & PHA & $418 \pm 88$ & $594 \pm 93$ \\
Autologous CD4+ & - & $542 \pm 47$ & $636 \pm 83$ \\
Allogeneic CD4+ & - & $692 \pm 95$ & $840 \pm 52$ \\
Autologous CD4+ & PHA & $774 \pm 104$ & $27,774 \pm 742$ \\
Allogeneic CD4+ & PHA & $611 \pm 83$ & $17,935 \pm 89$ \\
& & & \\
\hline
\end{tabular}

* Nonadherent, T cell-depleted (E-) lymphocytes $\left(1 \times 10^{5} /\right.$ well $)$ were cultured with mitomycin C-treated autologous or allogeneic CD4+ T cells $\left(7.5 \times 10^{4} /\right.$ well $)$ in the presence or absence of PHA for $3 \mathrm{~d}$. Cultures were pulsed with $\left[{ }^{3} \mathrm{H}\right] \mathrm{TdR}$ during the final 14-16 h of culture. Results are expressed as mean counts per minute uptake \pm SEM.

of BLS B cells was observed, even when cultures were allowed to incubate for 4-6 wk. Transformation of B cells from control individuals was uniformly successful.

$B$ cell differentiation to antibody-secreting cells. The ability of BLS B cells to differentiate into antibody-secreting plasma cells was assessed in a PWM assay. As shown in Table V, virtually no B cell differentiation was observed when BLS B cells were cultured in the presence of PWM-activated CD4+ cells; only a small amount of IgM was produced. This lack of response was seen using both allogeneic and autologous $T$ cells. Since the production of antibody in this assay is largely a function of in vivo-activated B cells (27), these results suggest that the defect in BLS B cell activation observed in vitro mirrors a comparable in vivo defect.

\section{Discussion}

The bare lymphocyte syndrome is a rare immunodeficiency disorder characterized by the absence of class I and/or class II membrane antigens encoded for by genes of the MHC. In the studies herein, we have examined the consequences of deficient expression of class II MHC antigens on the differentia-

Table V. B Cell Differentiation in the Bare Lymphocyte Syndrome

\begin{tabular}{llcr}
\hline & & \multicolumn{2}{c}{ Immunoglobulin secretion } \\
\cline { 3 - 4 } B cells* & CD4+ cells & IgM & IgG \\
\hline & & & $n g / m l$ \\
BLS & - & 125 & 100 \\
BLS & BLS & 175 & 100 \\
BLS & Control & 475 & 125 \\
Control & - & 225 & 450 \\
Control & BLS & 700 & 750 \\
Control & Control & 6,500 & 7,750
\end{tabular}

* Nonadherent, T cell-depleted (E-) lymphocytes $\left(7.5 \times 10^{4} /\right.$ well) were cultured with BLS or control CD4+ cells $\left(7.5 \times 10^{4} /\right.$ well $)$ and PWM for $7 \mathrm{~d}$. Culture supernatants were harvested and analyzed for IgM or IgG with an ELISA. Results are expressed as nanograms per milliliter of antibody. tion and functions of B lymphocytes. Our studies show that class II antigen-deficient $B$ cells have a profound intrinsic defect in their response to stimuli that induce B cell activation and proliferation. This functional defect is accompanied by an apparent block in B cell differentiation that results in the presence of a homogeneous population of B cells that uniformly express the HB-4 antigen, a marker normally found on only a subset of B cells. In addition, we show for the first time that these cells may also fail to express a non-MHC encoded B cell antigen, the CD21 (C3d/EBV receptor) molecule.

The precise molecular defects responsible for the MHC deficiency syndrome have not been identified. Since the syndrome can involve defective expression of class I, class II, or both class I and II antigens (5), it is likely that the defects responsible for this syndrome are heterogeneous. Although the BLS could result from deletion of the structural genes encoding for MHC antigens, this defect has not yet been reported, and hybridization studies with probes for class II genes demonstrated that all class II MHC structural genes were present in this patient (data not shown). A variety of regulatory gene defects could also produce the MHC deficiency syndrome, and defective transcription of class II MHC genes has been demonstrated in cells from some BLS patients (28). Interestingly, the phenotypic consequences of the underlying defect(s) may differ in $\mathrm{T}$ cells and $\mathrm{B}$ cells. In reports to date, $\mathrm{T}$ cells from all BLS patients have retained the class II-deficient phenotype after activation and culture in IL-2. Identical results were obtained for IL-2-dependent $\mathrm{T}$ cell lines generated from the patient described herein (data not shown). In contrast, the B cell defect in certain BLS patients can be partially overcome after in vitro transformation with EBV (28). Similarly, B cells from some BLS patients may express detectable HLA-DR antigens in vivo (29). Thus, it appears that the primary defect(s) may be incompletely expressed in B cells from at least some BLS patients. Furthermore, this may be associated with a mitigation of the functional deficits, since these patients produced antibody in vivo and in vitro (29). Although we were unable to obtain EBV-transformed B cells for analysis, the B cells from this patient did not express class II antigens when cultured with anti-mu antibodies, a stimulus that significantly increases class II antigen expression on normal B cells (30). Thus, it appears that the heterogeneity of the molecular lesions may produce variability in the magnitude of both the class II deficiency and the B cell functional impairment.

The early maturational events leading to the production of B cells do not appear to be affected by the absence of class II antigens since the numbers of sIg + cells are normal in the BLS. The vast majority of the B cells in the BLS were sIgM+/sIgD+ cells, as is seen in normal individuals and other BLS patients $(4,30)$. Furthermore, the sIgM appeared to be inserted in a functionally relevant fashion since cross-linkage with anti-mu antibodies provided the initial activation signal resulting in B cell enlargement. The expression of kappa and lambda light chains was also normal. The most notable abnormality regarding surface Ig expression observed was the absence of detectable sIgA+ B cells. It is unlikely that this represents physiological variability, since sIgA+ B cells are normally present in children of this age (31). This finding could reflect $(a)$ disruption, at any level, of the coordinated events involved in heavy chain isotype switching and sIg expression, or $(b)$ a defect in a helper $\mathrm{T}$ cell function required for the expansion of $\operatorname{sigA}+$ cells. 
Analyses of the expression of other B cell membrane antigens on class II-deficient B cells revealed several interesting findings. First, there was an increased frequency of B cells expressing the CD38 antigen in the BLS. This antigen is expressed by a population of relatively immature B cells (17). In conjunction with previous studies showing that BLS B cells retain a neonatal pattern of $\operatorname{sig} M$ and $\operatorname{sIgD}$ expression $(4,30)$, these data suggest that B cell maturation in the BLS may be delayed or arrested. This possibility was further supported by the observation that the B cells in the BLS uniformly expressed the HB-4 antigen. In normal adults and neonates, the HB-4 antigen is expressed by a subset of relatively immature (but functionally intact) resting $B$ cells (15). Although there are several possible explanations for the absence of the HB-4 antigen-negative subset, we believe this reflects an arrest in B cell maturation, perhaps analogous to that proposed to account for the deficiency of the Lyb5+ subset in xid mice (32). This arrest could be due to a primary B cell defect associated with the absence of class II MHC antigens, or it could be a secondary to deficient immunocompetent cell interactions.

The most striking phenotypic abnormality associated with the absence of class II MHC antigens was the absence of CD21 antigens. This membrane molecule has previously been shown to serve as the B cell receptor for the C3d complement fragment as well as for the $\operatorname{EBV}(23,24)$. It has also been suggested that this antigen may play a role in mediating signals associated with $B$ cell activation $(33,34)$. Thus, the absence of CD21 antigens may contribute to the B cell functional defects observed in our studies. This is particularly true with regard to our inability to transform the BLS B cells with EBV, since the receptor for virus is essential for transformation (35). Although EBV-transformed B cell lines from some BLS patients have been established $(28,36)$, analyses of CD21 antigen expression on B cells from these patients have not been reported. Given the apparent heterogeneity of this syndrome in other respects, it is possible that the expression of CD21 antigens may also vary quantitatively. However, it is also likely that class II-deficient $B$ cells are unable to respond appropriately to the mitogenic effects of EBV, in analogous fashion to their failure to respond to other B cell mitogens. The slow initial growth that has been reported for transformed BLS B cells (36) is consistent with the data from our functional studies.

The basis for the defect of CD21 antigen expression in this patient is currently unknown. In normal B cells, this antigen is expressed during the transition of pre-B cells to their sIg+ progeny, and it is normally present on the vast majority of sIg + cells in the neonate and adult (16). Thus, the absence of CD21 antigens could indicate that pre-B to $B$ cell maturation is incomplete in the BLS. Alternatively, this absence could result from the same regulatory defect responsible for the class II MHC antigen deficiency. Such a hypothetical molecular defect would have to be quite limited in nature, since the expression of the majority of B cell membrane antigens is unaffected. Moreover, the expression of these molecules does not appear to be inherently linked, since mutant B cell lines deficient in class II MHC antigen expression do express CR2 molecules reactive with the HB-5 mab (Cotner, T., and L. T. Clement, unpublished observations).

In addition to the defect in $B$ cell proliferation, this patient's B cells were also deficient in their ability to differentiate in a pokeweed mitogen assay to become immunoglobulin-secreting cells, thus mimicking the patient's hypogammaglobulinemia in vivo. This was true whether the B cells were cul- tured with purified autologous CD4+ helper cells or with those from a normal control donor. The B cells that respond in this $T$ cell-dependent assay are large, low density sIgD- cells that presumably have been previously activated in vivo (27). Since BLS B cells appear to be intrinsically abnormal in this regard, one would not anticipate their presence in the circulation. It should also be noted, however, that the helper $T$ cells in the BLS patient failed to induce differentiation of normal B cells, although they were capable of inducing normal B cells to proliferate. Whereas quantitative defects in CD4+ $\mathrm{T}$ cells have been described in several other BLS patients, the observed helper cell defect was not merely quantitative because purified CD4+ cells were used. The basis for this defect is currently under investigation.

Since class II MHC antigens mediate interactions among immunocompetent cells that are required for B cell differentiation and antibody production, it has generally been assumed that the hypogammaglobulinemia seen in BLS patients results from ineffective interactions among $T$ cells, $B$ cells, and accessory cells. However, recent studies have suggested that the role of these membrane molecules in the functions of $B$ cells is not restricted to regulating cellular interactions. Rather, it appears that class II MHC antigens are integral participants in the intracellular processes associated with B cell activation $(9-12,37,38)$, events that are quite independent of $\mathrm{T}$ cell or accessory cell influences. We have previously shown that monoclonal antibodies reactive with human class II antigens significantly inhibited stimulus-induced increases in B cell RNA synthesis and proliferative responses to $T$ cell-dependent or $\mathrm{T}$ cell-independent stimuli. In contrast, these antibodies only partially inhibited activation-dependent increases in B cell size. Although these data suggested that class II MHC molecules participate in early, inductive events of the B cell activation cascade, the use of exogenous antibodies complicated the interpretation of these data, since anti-class II antibodies might produce a negative signal that directly inhibited or otherwise disturbed cellular activation. Since we have shown in the present studies that B lymphocytes deficient in their expression of class II MHC antigens have virtually identical abnormalities in activation and proliferation as those effected by anti-class II antibodies, it is reasonable to infer that the previously described effects of anti-DR and anti-DQ antibodies were the result of alterations in the functions and/or intracellular traffic of class II molecules rather than secondary to an externally-imposed negative signal. Thus, this "experiment of nature" adds important support for the notion that class II antigens participate directly in the physiology of B cell activation.

\section{Acknowledgments}

Supported by U. S. Public Health Service grants CA-42735, CA-12800, CA-12375, AI-15332, and AI-15251, awarded by the National Institutes of Health.

\section{References}

1. Touraine, J.-L., H. Betuel, G. Gouillet, and M. Jeune. 1978. Combined immunodeficiency disease associated with absence of cellsurface HLA-A and -B antigens. J. Pediatr. 93:47-51.

2. Schuurman, R. K. B., J. J. van Rood, J. M. Vossen, P. T. A. Schellekens, T. M. Feltkamp-Vroom, E. Doyer, F. Gmelig-Meyling, and H. K. A. Visser. 1979. Failure of lymphocyte-membrane HLA-A and -B expression in two siblings with combined immunodeficiency. Clin. Immunol. Immunopathol. 14:418-434. 
3. Griscelli, C., A. Durandy, and J. L. Virelizier. 1980. Impaired cell to cell interaction in partial combined immunodeficiency with variable expression of HLA antigens. In Primary Immunodeficiencies. M. Seligmann and W. H. Hitzig, editors. Elsevier/North Holland, Amsterdam. 499-503.

4. Hadam, M. R., R. Dopfer, G. Dammer, H.-H. Peter, M. Schlesier, C. Muller, and D. Niethammer. 1984. Defective expression of HLA-DR region determinants in children with congenital agammaglobulinemia and malabsorption: A new syndrome. In Histocompatibility Testing 1984. E. D. Albert, M. P. Baur, and W. R. Mayr, editors. Springer-Verlag, Heidelberg, FRG. 645-650.

5. Touraine, J.-L., G.-L. Marseglia, and H. Betuel. 1985. Thirty international cases of bare lymphocyte syndrome. Biological signifcance of HLA antigens. Exp. Hematol. (Suppl. 17) 13:86-87.

6. McDevitt, H. O. 1976. Functional analysis of Ia antigens in relation to genetic control of the immune response. In The Role of the Products of the Histocompatibility Gene Complex in Immune Responses. D. H. Katz and B. Benacerraf, editors. Academic Press, New York. 257.

7. Benacerraf, B. 1981. Role of MHC gene products in immune regulation. Science (Wash. DC). 212:1229-1235.

8. Schwartz, R. H. 1984. The role of gene products of the major histocompatibility complex in $\mathrm{T}$ cell activation and cellular interactions. In Fundamental Immunology. W. E. Paul, editor. Raven Press, New York. 379.

9. Niederhuber, J. E., J. A. Frelinger, E. Dugan, A. Coutinho, and D. C. Shreffler. 1975. Effects of anti-Ia serum on mitogenic responses. Inhibition of the proliferative response to B cell mitogen, LPS, by specific anti-Ia sera. J. Immunol. 115:1672-1677.

10. Forsgren, S., G. Pobor, A. Coutinho, and M. Pierres. 1984. The role of I-A/E molecules in B lymphocyte activation. I. Inhibition of lipopolysaccharide-induced responses by monoclonal antibodies. $J$. Immunol. 133:1204-1210.

11. Clement, L. T., T. F. Tedder, and G. L. Gartland. 1986. Antibodies reactive with class II antigens encoded for by the major histocompatibility complex inhibit human B cell activation. J. Immunol. 136:2375-2381.

12. Bonagura, V. R., N. Agostino, M. K. Crow, and B. Pernis. 1985. Anti-immunoglobulin stimulation of human B lymphocytes is inhibited by anti-class II major histocompatibility complex antibodies. Cell. Immunol. 96:442-447.

13. Haas, A., S. P. Marshall, T. Chin, J. McKinney-Clark, B. J. Ank, J. Giorgi, P. Terasaki, L. T. Clement, and E. R. Stiehm. 1986. Immunodeficiency with absent class II HLA antigens: In vitro induction by gamma-interferon (IFN). Pediatr. Res. 20:294. (Abstr.)

14. Landay, A., G. L. Gartland, and L. T. Clement. 1983. Characterization of a phenotypically distinct subpopulation of Leu-2+ cells that suppresses $T$ cell proliferative responses. J. Immunol. 131:27572761.

15. Tedder, T. F., L. T. Clement, and M. D. Cooper. 1985. Development and distribution of a human B cell subpopulation identified by the HB-4 monoclonal antibody. J. Immunol. 134:1539-1544.

16. Tedder, T. F., L. T. Clement, and M. D. Cooper. 1984. Expression of $\mathrm{C} 3 \mathrm{~d}$ receptors during human $\mathrm{B}$ cell differentiation: Immunofluorescence analysis with the HB-5 monoclonal antibody. J. Immunol. 133:678-683.

17. Tedder, T. F., L. T. Clement, and M. D. Cooper. 1984. Discontinuous expression of a membrane antigen (HB-7) during B lymphocyte differentiation. Tissue Ant. 24:140-151.

18. Dianzani, U., M. Massaia, A. Pileri, C. E. Grossi, and L. T. Clement. 1986. Differential expression of ecto- 5 ' nucleotidase activity by functionally and phenotypically distinct subpopulations of human Leu 2+/T8+ lymphocytes. J. Immunol. 137:484-489.

19. Sherr, E. H., L. D. Stein, H.-M. Dosch, and A. Saxon. 1987. IgE enhancing activity directly and selectively affects activated B cells. Evidence for a human IgE differentiation factor. J. Immunol. 138:3836-3843.

20. Maruyama, S., H. Kubagawa, and M. D. Cooper. 1985. Acti- vation of human $B$ cells and inhibition of their terminal differentiation by monoclonal anti-mu antibodies. J. Immunol. 135:192-199.

21. Clement, L. T., M. K. Dagg, and G. L. Gartland. 1984. Small, resting B cells can be induced to proliferate by direct signals from activated helper T cells. J. Immunol. 132:740-744.

22. Clement, L. T., C. E. Grossi, and G. L. Gartland. 1984. Morphologic and phenotypic features of the subpopulation of Leu-2+ cells that suppresses B cell differentiation. J. Immunol. 133:2461-2468.

23. Tedder, T. F., J. J. Weis, L. T. Clement, D. F. Fearon, and M. D. Cooper. 1986. The role of receptors for complement in the induction of polyclonal B cell proliferation and differentiation. J. Clin. Immunol. 6:65-73.

24. Fingeroth, J. D., J. J. Weis, T. F. Tedder, J. L. Strominger, P. A. Biro, and D. T. Fearon. 1984. Epstein-Barr virus receptor of human B lymphocytes is the C3d receptor (CR2). Proc. Natl. Acad. Sci. USA. 81:4510-4517.

25. DeFranco, A. L., E. S. Raveche, R. Asofsky, and W. E. Paul. 1982. Frequency of B lymphocytes responsive to anti-immunoglobulin. J. Exp. Med. 155:1523-1532.

26. Muraguchi, A., J. L. Butler, J. H. Kehrl, and A. S. Fauci. 1983. Differential sensitivity of human B cell subsets to activation signals delivered by anti-mu antibody and proliferative signals delivered by a monoclonal B cell growth factor. J. Exp. Med. 157:530-541.

27. Kuritani, T., and M. D. Cooper. 1982. Human B cell differentiation. II. Pokeweed mitogen-responsive B cells belong to a surface immunoglobulin D-negative subpopulation. J. Exp. Med. 155:15611565.

28. Lisowska-Grospierre, B., D. J. Charron, C. de Preval, A. Durandy, C. Griscelli, and B. Mach. 1985. A defect in the regulation of major histocompatibility complex class II gene expression in human HLA-DR negative lymphocytes from patients with combined immunodeficiency syndrome. J. Clin. Invest. 76:381-385.

29. Rijkers, G. T., J. J. Roord, F. Koning, W. Kuis, and B. J. M. Zegers. 1987. Phenotypical and functional analysis of B lymphocytes of two sibling with combined immunodeficiency and defective expression of major histocompatibility complex (MHC) class II antigens on mononuclear cells. J. Clin. Immunol. 7:98-106.

30. Monroe, J. B., and J. C. Cambier. 1983. B cell activation. III. B cell plasma membrane depolarization and hyper-Ia antigen expression induced by receptor immunoglobulin crosslinking are coupled. J. Exp. Med. 158:1589-1601.

31. Gathings, W. E., H. Kubagawa, and M. D. Cooper. 1981. A distinctive pattern of B cell immaturity in perinatal humans. Immunol. Rev. 57:107-133.

32. Smith, H. R., L. J. Yaffe, D. L. Kastner, and A. D. Steinberg. 1986. Evidence that Lyb-5 is a differentiation antigen in normal and xid mice. J. Immunol. 136:1194-1200.

33. Nemerow, G. R., R. Wolfert, M. McNaughton, and N. R. Cooper. 1985. Identification of the Epstein-Barr virus receptor and its relationship to complement C3d receptor (CR2). Fed. Proc. 44:988.

34. Wilson, B. S., J. L. Platt, and N. E. Kay. 1985. Stimulation of B cell DNA synthesis by monoclonal antibodies to the CR2 (C3d) complement fragment receptor. Fed. Proc. 44:1876.

35. Volsky, D. J., I. M. Shapiro, and G. Klein. 1980. Transfer of Epstein-Barr virus receptors to receptor-negative cells permits virus penetration and antigen expression. Proc. Natl. Acad. Sci. USA. 77:5453-5457.

36. Hadam, M. R., C. de Preval, R. Dopfer, G. Dammer, H.-H. Peter, B. Mach, and D. Niethammer. 1985. Deficiency of HLA-D region antigens in patients with congenital agammaglobulinemia and malabsorption. Studies with lymphoblastoid cell lines established from patients. In Workshop on Primary Immunodeficiency Diseases. M. M. Eibl and F. S. Rosen, editors. Excerpta Medica, New York. 19.

37. Pletscher, M., and B. Pernis. 1983. Internalized membrane immunoglobulin meets intracytoplasmic DR antigen in human B lymphoblastoid cell lines. Eur. J. Immunol. 13:581-587.

38. Tse, D. B., and B. Pernis. 1984. Mobilization of membrane I-A upon membrane IG cross-linkage and internalization in B cells. Fed. Proc. 43:1422. 